\title{
Editorial
}

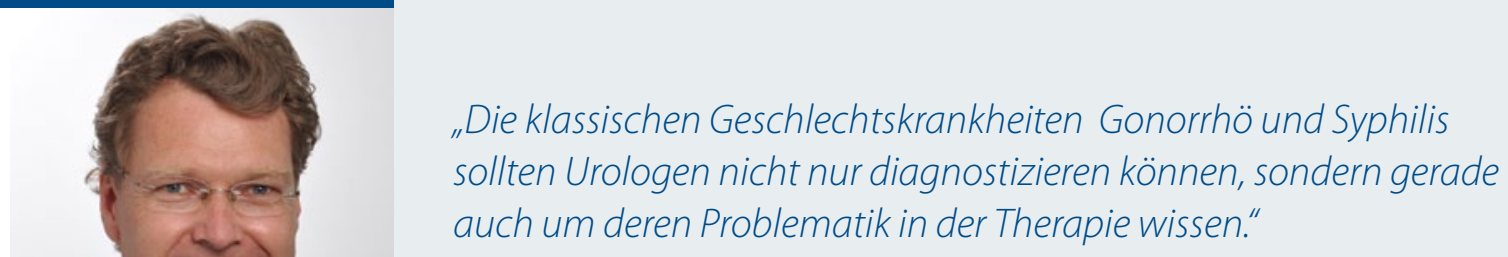

Prof. Dr. med. Peter Schneede,

Urologische Klinik, Klinikum Memmingen

\section{Aufklärungsarbeit eminent wichtig}

$\mathrm{M}$ it den Jahrestagungen 2003 (München) und 2010 (Memmingen) hat die Deutsche STD-Gesellschaft (DSTDG) wiederholt die Tagungspräsidentschaft in die Hände eines Urologen gelegt und damit ihr großes Interesse an urologischer Mitarbeit in der interdisziplinär besetzten Gesellschaft demonstriert. Tatsächlich sind nicht allein jüngere, wissenschaftlich interessierte Urologinnen und Urologen, sondern auch vorwiegend klinisch tätige und niedergelassene Kollegen in der DSTDG herzlich willkommen.

\section{Urologen als Ansprechpartner}

Die vorliegende Ausgabe der URO-NEWS beschäftigt sich in ihrem Schwerpunkt nochmals mit dem Thema der sexuell übertragbaren Erkrankungen (STDs), die trotz aller Bemühungen von DGU und BDU unter Urologen nicht immer ausreichend Beachtung gefunden haben. Gerade in den letzten Jahren hat sich aber vieles auf diesem Gebiet verändert, sodass URO-NEWS die wichtigsten Neuerungen durch ausgewiesene Experten für seine Leser nochmals zusammenfassen lassen möchte. Schließlich werden Urologen alltäglich wegen Infektionen der Harnund Geschlechtsorgane konsultiert. Die klassischen Geschlechtskrankheiten Gonorrhö und Syphilis sollten Urologen deshalb nicht nur diagnostizieren können, sondern gerade auch um deren Problematik in der Therapie bedingt durch aktuelle Resistenzentwicklungen oder durch Ko-Infektionen mit anderen STDs wissen. HPV-Erkrankungen, wie beispielsweise die zu Rezidiven neigenden Kondylome oder HPV-assoziierte Genitaltumore beiderlei Geschlechts, können heute wirkungsvoll durch Präventivimpfungen verhindert werden. Betroffene Patienten und Eltern noch zu schützender Kinder und Jugendlicher fragen heute gezielt auch Urologen nach Impfmöglichkeiten. Verwirrend widersprüchliche Berichterstattungen in der Presse aber auch eine nicht zu unterschätzende Impfmüdigkeit beziehungsweise eine wachsende Anzahl von Impfgegnern in der Bevölkerung könnten andererseits in Deutschland den Erfolg der HPV-Impfung nachhaltig infrage stellen, der sich in den Ländern mit genereller Schulimpfung bereits wenige Jahre nach Einführung der HPV-Impfprogramme deutlich abzeichnet. Hier ist dringlich weitere Aufklärungsarbeit erforderlich.

\section{Klassische STDs breiten sich immer wieder aus}

Leider ist die Impfstoffentwicklung gegen andere sexuell übertragbare Virusinfektionen (z.B. Herpes simplex-Virusinfektionen) bislang weniger erfolgreich. Dennoch wurden auch bei HIV-Infektionen große Therapie-Fortschritte erzielt, die es heute ermöglichen, die HIV-Krankheit wesentlich besser und vor allem langfristiger kontrollieren zu können. Dadurch ist die Lebenserwartung HIVInfizierter nicht wesentlich schlechter als diejenige der nicht infizierten Normalpopulation. Auch diese Tatsache mag dazu beigetragen haben, dass gerade in sexuellen Risikogruppen, insbesondere unter Männern, die Sex mit Männern haben (MSM), Vorsichtsmaßnahmen nachgelassen haben. Die klassischen Geschlechtskrankheiten konnten sich so in den letzten Jahren endemisch immer wieder ausbreiten.

Urologen, die sich über die Beiträge in diesem Heft hinaus über sexuell übertragbare Infektionen und Erkrankungen informieren möchten, sollten regelmäßig die Homepage des Robert Koch-Instituts (www.rki.de) besuchen oder sich die nächste Jahrestagung der DSTDG vom 1. bis 3. September 2011 in Frankfurt am Main vormerken. Auf diesem nationalen Kongress wird umfassend über alle Neuerungen zum Thema STD aber auch zum Thema Sexuelle Gesundheit berichtet.

Ihr

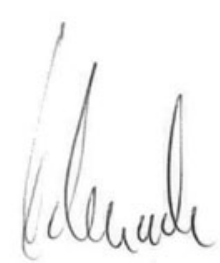

